\title{
Prevention of Lateral Elbow Tendinopathy
}

\author{
Stasinopoulos Dimitrios \\ Physiotherapy Program, Department of Health Sciences, School of Sciences, European University Cyprus, Director of Cyprus Musculoskeletal \\ and Sports Trauma Research Centre (CYMUSTREC), Cyprus \\ *Corresponding Author: Stasinopoulos Dimitrios, Physiotherapy Program, Department of Health Sciences, School of Sciences, European \\ University Cyprus, Director of Cyprus Musculoskeletal and Sports Trauma Research Centre (CYMUSTREC), Cyprus.
}

Received: July 19, 2019; Published: August 01, 2019

Lateral elbow tendinopathy (LET) is one of the two most common tendinopathies of the upper extremity among athletes and non-athletes. LET is usually defined as a syndrome of pain in the area of the lateral epicondyle which may be degenerative rather than inflammatory. The main complaints of patients with LET are pain and decreased function both of which may affect daily activities. A plethora of physiotherapy techniques, electrotherapeutic and non-electrotherapeutic modalities, has been recommended for the management of LET. Although the patients with LET will seek physiotherapy treatment or modify training, many of them will experience recurrent symptoms and a few of them may stop daily activities due to persistent pain. Therefore, it is of interest to identify ways to prevent LET so «patients» never enter a cycle of recurrent pain and functional limitation.

There are four stages to injury prevention outlined by van Mechelen., et al. [1].These include 1) establishing the extent of the injury problem; 2) establishing the etiology and mechanisms of injury; 3) introducing preventative measures; and 4) assessing the effectiveness of these measures by reassessing the extent of the problem. Identification of risk factors (stage 2) for LET is essential to the development of targeted prevention programmes. It is commonly accepted that LET is the effect of overuse, over-stress or over-exertion of the extensor tendons of the wrist, especially Extensor Carpi Radialis Brevis (ECRB), the most commonly affected tendon in LET by quick, continuously monotonous, repetitive and/ or strenuous activities of the wrist. If this were the only cause of LET, it would beg the question: why do researchers not face this cause and establish an effective prevention program for LET? The answer is simple: the etiology of LET remains relatively unknown and unexplored because LET is a multifactorial condition in nature and, although the overuse of the wrist is the main cause, it is not the only one [2]. Other factors that play a significant role in the etiology of LET can be previous injury, age, sex, poor vascular supply of ECRB, anatomical variation such as differences in alignment and range of motion, decreased flexibility, decreased eccentric muscle strength, activity/ training volume, technique, changes in pain pathways and the motor system and cervical spine dysfunction [2].However, no studies were found to show a relation between LET and the above referred causes. Overall, a determination of the causes of LET requires further research since, if clinicians were to understand the etiology of LET, firstly an effective treatment for LE might be more easily established and secondly LET might be more easily prevented [2].

The above mentioned risk factors are classified as intrinsic (within the body) or extrinsic (outside the body) and modifiable or non-modifiable. However, many recommended risk factors have not been prospectively investigated, have limited support from low-quality studies or have conflicting evidence. Additionally, not all risk factors were modifiable. Thus, they may assist in identifying patients at higher risk of LET but do not provide an opportunity for clinicians to intervene to reduce that risk.

No randomized trials have evaluated preventative interventions for LET. Preventive measures (Table 1) have been recommended in the practice3, but further research is needed to bridge the gap between research and practice, since it is important the effective preventive interventions be translated into successful implementation [4].

\begin{tabular}{|l|}
\hline Stretching exercises \\
\hline Strengthening exercises /loading program \\
\hline Proprioceptive exercises \\
\hline External support (taping/bracing/kinesiotaping) \\
\hline Soft tissue techniques \\
\hline Relative rest / Avoid Repetitive Tasks / Take breaks \\
\hline Ideal rehabilitation program of previous injuries \\
\hline Correction of sports technique / activity \\
\hline
\end{tabular}

Table 1: Recommended preventive measures for LET.

The below factors have to be considered in order to investigate preventative interventions for LET:

1. Primary vs secondary prevention5. Primary prevention is aimed at preventing an injury before it occurs. In contrast to primary prevention, secondary prevention aims to reduce the impact of an injury that has already occurred and to prevent re-injury. 
2. Tendon normalization and patients' symptoms6. Patients asymptomatic with abnormalities, patients asymptomatic without abnormalities, patients symptomatic with abnormalities and patients symptomatic without abnormalities will follow the same prevention program?

3. Most risk factors are not strongly associated with injury so do not clearly delineate individuals who will develop injury from those who do not. Therefore, preventative interventions may be more effective if applied across an entire cohort rather than solely among individuals deemed to be at greater risk of injury [7].

4. Definition of injury and injury incidence [8]

5. Prevention should be focused on multiple risk factors that af fect a specific individual, because the etiology of LET is multifactorial [6].

6. Individualize / modify the prevention program according to tendon abnormality, etiology and previous injury

7. The current challenge to researchers is to identify prospective risk factors and then investigate whether modifying these factors reduces injury risk in cohorts of individuals at risk of tendinopathy [1]

\section{Bibliography}

1. Van Mechelen W., et al. "Incidence, severity, aetiology and prevention of sports injuries". Sports Medicine 14.2 (1992): 82-99.

2. Stasinopoulos D. "The Difficulty of Establishing Effective Treatments for Lateral Elbow Tendinopathy". Biomedical Journal of Scientific and Technical Research 6 (2018): 1-4.

3. Hume PA., et al. "Epicondylar injury in sport: epidemiology, type, mechanisms, assessment, management and prevention". Sports Medicine 36.2 (2006): 151-170.

4. Verhagen E., et al. "A knowledge transfer schemeto bridge the gap between science and practice: an integration of exist-ing research frameworks into a tool for practice". British Journal of Sports Medicine 48.8 (2014): 698-701.

5. Ingrid Barelds., et al. "Ankle Bracing is Effective for Primary and Secondary Prevention of Acute Ankle Injuries in Athletes: A Systematic Review and Meta-Analyses". Journal of Sports Medicine 48.12 (2018): 2775-2784.
6. Peters JA., et al. "Preventive interventions for tendinopathy: A systematic review". Journal of Science and Medicine in Sport 19.3 (2016): 205-211.

7. Malliaras P and O'Neil S. "Potential risk factors leading to tendinopathy". Apunts Medicine Esport 52 (2017): 71-77.

8. Sprague AL., et al. "Modifiable risk factors for patellar tendinopathy in athletes: a systematic review and meta-analysis". British Journal of Sports Medicine 52.24 (2018): 1575-1585.

\section{Volume 2 Issue 9 September 2019 \\ (C) All rights are reserved by Stasinopoulos Dimitrios.}

